\title{
Una mirada a la equidad en nuestras prácticas desde la dimensión política de la educación matemática
}

A Look at Equity in our Practices from the Political Dimension of Mathematics Education

Um olhar sobre a equidade nas nossas práticas desde a dimensão política da educação matemática

\section{Francisco Javier Camelo-Bustos* (iD o000-0002-8627-4816 Gabriel Mancera-Ortiz** (iD 0000-0003-3556-9283 \\ Claudia Salazar-Amaya*** (iD) 0000-0003-0147-0349}

\author{
Artículo de reflexión \\ Revista Colombiana de Educación, N.ํ73. Segundo semestre de 2017, Bogotá, Colombia. \\ doi: http://dx.doi.org/10.17227/01203916.73rce281.298 \\ Para citar este artículo: Camelo, F., Mancera, G., y Salazar C. (2017). Una mirada a la equidad en \\ nuestras prácticas desde la dimensión política de la educación matemática. Revista Colombiana \\ de Educación, (73), 283-300.
}

\section{(c) $\underset{\mathrm{BY}}{\mathrm{B}}(\mathrm{SC}$}

* Estudiante de doctorado en Educación. Profesor de la Universidad Distrital Francisco José de Caldas. Bogotá, Colombia. Correo electrónico: ficamelob(a)gmail.com

** Estudiante de doctorado en Educación. Profesor de la Universidad Distrital Francisco José de Caldas. Bogotá, Colombia.

*** Estudiante del Doctorado Interinstitucional en Educación. Profesora de la Universidad Pedagógica Nacional. Bogotá, Colombia.. 


\title{
Resumen
}

En este documento nos hemos propuesto problematizar el significado de equidad desarrollado desde planteamientos teóricos del campo de la educación, para contribuir a la reflexión sobre la equidad en la educación matemática. Para llevar a cabo tal propósito ejemplificamos, a la luz de las tres dimensiones que configuran dicho concepto (acceso, permanencia y éxito) cómo, desde nuestras prácticas pedagógicas e investigativas en educación matemática, hemos contribuido a promover la equidad. policies. Lo anterior nos permitió cuestionar, en cierta medida, cómo el sistema educativo colombiano ha promovido, a través de la política educativa, mecanismos para una falsa equidad. Nuestras afirmaciones se sustentan en tres asuntos: (i) el acceso se ha considerado desde la perspectiva de aumento de cobertura pero no ha implicado el reconocimiento de quienes son los niños y jóvenes que ingresan al sistema; (ii) la permanencia se pretende conseguir aumentando el tiempo de la escolaridad pero no ha implicado la generación de ambientes de aprendizaje, que promuevan la participación de niños y jóvenes, en prác ticas significativas con las matemáticas, que aporten a la construcción de su proyecto de vida; (iii) el éxito ha sido considerado en términos de logros individuales sin considerar la importancia de promover comprensiones colectivas y acciones cooperativas que conlleven la superación de problemáticas sociales. En definitiva, planteamos que la manera en la que la política ha promovido la "equidad" no garantiza prácticas escolares en las que se constituyan subjetividades e intersubjetividades que favorezcan posibilidades de ser y actuar con otros

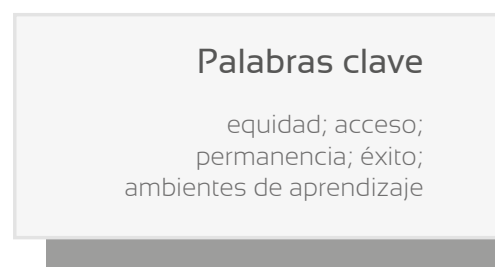

\section{Keywords}

equity; access; permanence; learning environments

\begin{abstract}
In this document we propose a challenge to the meaning of equality from the theoretical framework of the field of education, seeking to promote a reflection about equality in mathematics education. To that end, we rely on the three dimensions that constitute the concept of equality (access, retention rates, and success) to illustrate how we have contributed to promote equality using our pedagogic and investigative practices in mathematics education. This approach has allowed us to question, to some extent, how the policies of the Colombian education system have promoted the mechanisms for a false equality. Our assertions rely on three key issues: (i) access has been considered mostly from the perspective of increased coverage, without a clear understanding of who the children and the youths admitted into the system really are; (ii) retention rates are sought by a mere extension of the permanence in the school programs, without the generation of a learning environment that promotes the participation of children and youths in a meaningful use of mathematics that helps them advance their life projects; (iii) success has been understood in terms of individual achievement without considering the importance of promoting collective comprehension and cooperative actions that provide skills to overcome social problems. In summary, we argue that the existing policies to promote "equality" do not guarantee school practices that promote subjectivities and inter-subjectivities that nurture the possibilities of being and working with others
\end{abstract}

\begin{abstract}
Resumo
Neste documento, visamos problematizar o significado de equidade desenvolvido desde abordagens teóricas do campo da educação, para contribuir à reflexão sobre a equidade na educação matemática. Para efetuar este propósito, exemplificamos, com base nas três dimensões que compõem esse conceito (Acesso, permanência e sucesso) como, desde nossas práticas pedagógicas e pesquisas em educação matemática, contribuímos para promover a equidade. Isso permitiu questionar, em certa medida, como o sistema educativo colombiano promove, através da política educativa, mecanismos para uma falsa equidade. Nossas afirmações estão sustentadas em três assuntos: (i) o acesso foi considerado desde a perspectiva do aumento de cobertura, mas não implica o reconhecimento que quais são as crianças e jovens que ingressam ao sistema; (ii) procura-se alcançar a permanência aumentando o tempo de escolaridade, mas não implica a geração de ambientes de aprendizagem que promovam a participação de crianças e jovens em práticas significativas com as matemáticas, que possam contribuir à constituição do seu projeto de vida; (iii) o sucesso é considerado como conquistas individuais sem considerar a importância de promover compreensões coletivas e ações cooperativas que impliquem a superação de problemáticas sociais. Em conclusão, consideramos que a forma na que a política promove a equidade não garante práticas escolares que possam constituir subjetividades e intersubjetividades que ofereçam possibilidades de ser e atuar com outros.
\end{abstract} N. ${ }^{\circ} 73$

\section{Palavras chave}

equidade; acesso permanência; sucesso; ambientes de aprendizagem 
Cuando las leyes de la matemática se refieren a la realidad, no son ciertas; cuando son ciertas, no se refieren a la realidad.

Albert Einstein

Cuando recibimos la invitación del comité editorial de la Revista Colombiana de Educación para escribir un artículo que reflexionara sobre asuntos de equidad, algunos integrantes del colectivo de investigación Edumadys ${ }^{1}$ asumimos el reto de repensarnos y revisar nuestras prácticas, con el propósito de dar cuenta de posibles conexiones entre asuntos de equidad y lo que hemos venido desarrollando en nuestras prácticas pedagógicas e investigativas en casi una década. El propósito fundamental de nuestro trabajo puede sintetizarse en la búsqueda de posibilidades para desarrollar tanto investigación como prácticas pedagógicas que incorporen el giro político de la educación matemática (García, Valero y Mancera, 2008 y García et al., 2009). Es decir, prácticas e investigaciones que centran la atención en torno a la comprensión y las posibilidades de abrir espacios para entender el conocer reflexivo -en el sentido de Skovsmose (1994)-, que se pueda generar en los estudiantes cuando ellos reflexionan, plantean y resuelven situaciones no matemáticas con las matemáticas, más que en algún tipo de desarrollo cognitivo. Las preguntas las centramos, entonces, en asuntos de cómo el poder y los contextos determinan lo que los estudiantes aprenden, cómo lo aprenden y para qué.

Esta tarea de relacionar los resultados de nuestra experiencia y la idea de equidad en educación matemática no resultó sencilla, aunque presentíamos que en nuestros trabajos encontraríamos tales conexiones y podríamos ejemplificarlas, incluso de tal modo que fueran ejemplarizantes para poder pensar acerca de lo que significa la equidad en el marco de las prácticas educativas en general y de las matemáticas en particular.

Las dificultades más sobresalientes para dar cuenta de este reto comenzaron cuando decidimos abordar el tratamiento de este concepto en la educación en general, para luego problematizarlo en nuestras prácticas en educación matemática en particular. Tal conceptualización implicaba interpretaciones de lo que significa acceder al derecho a la educación y sus relaciones con el tipo de sociedad que deseamos construir. En este sentido, y teniendo como objetivo para este artículo socializar cómo pensar la equidad en nuestras prácticas, decidimos comenzar este documento presentando algunos planteamientos desde la educación en general sobre lo que podría interpretarse como equidad, y cómo hemos asumido una interpretación de esta idea desde una visión política de la educación matemática. A partir de

1 El colectivo de investigación interinstitucional Educación Matemática, Diversidad y Subjetividades está conformado por educadores matemáticos de las universidades Pedagógica Nacional y Distrital Francisco José de Caldas. Hace parte del grupo de investigación Didáctica de la Matemática. 
ello, describimos cómo problematizamos las ideas de acceso, permanencia y éxito para dar cuenta de nuestro tratamiento a la equidad, usando como ejemplos las experiencias logradas en nuestros trabajos.

\section{Para pensar la equidad, "un poco de miel no basta"2}

A los miembros del colectivo Edumadys una continua lucha por el derecho que tenemos a la educación nos empuja día a día a superar una visión de la sociedad que nos coloca como víctimas pasivas de la segregación. No desconocemos con ello, en nuestra historia, los movimientos sociales, sus resistencias y las afirmaciones como sujetos de derecho (a la educación, por ejemplo). En este sentido pensar en lo que significa el derecho a la educación, ineludiblemente nos conduce a pensar en nuestra perspectiva acerca de los derechos. Por ello retomamos algunos de los planteamientos de Arroyo (2015, p. 19, citando a Santos y Chauí, 2013) menciona que

La hegemonía de los derechos humanos como lenguaje de dignidad humana es hoy indiscutible. Sin embargo, esta hegemonía convive con una realidad perturbadora. La gran mayoría de la población mundial no es sujeto de derechos humanos. Es objeto de discursos de derechos humanos. Debe, pues, comenzar por preguntarse si los derechos humanos sirven eficazmente a la lucha de los excluidos, de los explotados y de los discriminados o si, por el contrario, se torna más difícil. En otras palabras ¿será que la hegemonía de que goza hoy el discurso de los derechos humanos [es] el resultado de una victoria histórica o, por el contrario, de una derrota histórica? Sin embargo, cualquiera que sea la respuesta dada a esas preguntas, la verdad es que, siendo los derechos humanos el lenguaje hegemónico de la dignidad humana, ellos son insuperables, y los grupos sociales oprimidos no puede dejar de preguntar si los derechos humanos, incluso si una parte de la misma hegemonía que consolida y legitima su opresión, no podrán ser usados para subvertir. O sea ¿podrán los derechos humanos ser usados de modo contra-hegemónico?

Con estos presupuestos, resaltamos que el derecho a la educación enfrenta esperanzas, posibilidades y retos propios de los derechos humanos. No en vano los movimientos sociales, como lo señala Arroyo (2015), han sido persistentes en demandar el derecho a la educación de manera conjunta con otros derechos fundamentales (como la vida, el trabajo, el territorio, la identidad, la igualdad, la diversidad y la justicia, entre otros), a la vez que los discursos políticos declaran la importancia de la educación como instrumento para la constitución de nuevas realidades

2 Este título está inspirado en la canción Té para tres, interpretada por el grupo Soda Stereo. 
sociales, como se expresa en el Plan Nacional de Desarrollo 2014-2018 Todos por un Nuevo País, en su artículo 3, "Pilares del Plan Nacional de Desarrollo" del Gobierno nacional de Colombia:

El Plan asume la educación como el más poderoso instrumento de igualdad social y crecimiento económico en el largo plazo, con una visión orientada a cerrar brechas en acceso y calidad al sistema educativo, entre individuos, grupos poblacionales y entre regiones, acercando al país a altos estándares internacionales y logrando la igualdad de oportunidades para todos los ciudadanos. (Congreso de la República de Colombia, 2015, p. 1).

No obstante, como profesores nos corresponde ser cuidadosos con la lectura, interpretación y puesta en marcha de planteamientos como el anterior, toda vez que, como lo advierte Arroyo (2015), debemos superar, por una parte, la visión ingenua según la cual el derecho a la educación es el primero de los derechos y el que resulta ser garantía de los otros derechos, comprendiendo que en él confluyen una red de prácticas y estructuras sin las cuales éste no es posible. Por otra, la idea de que el derecho a la educación acontecerá como un proceso social y político aislado de los derechos humanos más básicos. De igual modo hemos comprendido que los derechos humanos, en particular el de la educación, no son individuales, pues los movimientos por la defensa de los derechos humanos son luchas colectivas que van en contravía del patrón de poder-saber que segrega e invisibiliza a ciertos colectivos de clase y de raza (Arroyo, 2015).

Reconocemos que desde este discurso se atribuye a la sociedad, el Estado y el derecho la tarea de atenuar las desigualdades y buscar la igualdad con mecanismos que implican acciones desiguales niveladoras. En este sentido, se considera que estas acciones implican garantizar más posibilidades a quienes menos han tenido y más necesitan, lo que lleva a pensar que la igualdad no es uniformidad, impone el reconocimiento de las diferencias (Bracho y Hernández, 2009). Lo interesante de este sentido atribuido a la igualdad es la identificación del sujeto para evitar la injusticia inherente a la uniformidad, pues la diferencia se instaura como una condición del sujeto y no solo se establece en términos de variables como privilegio económico, social o de clase. Así, la igualdad requiere reconocimiento y equilibrio de las diferencias; implica la búsqueda de unidad en la diversidad.

Este sentido atribuido a la igualdad requiere pensar, como ya lo advertía la crítica de Popper a los planteamientos de Platón, que determinar lo que corresponde a cada quien no depende solo del lugar que ocupa el sujeto en la estructura piramidal de la sociedad, sino que se determina de acuerdo con la necesidad de cada ser humano en relación con los 
valores, fines y objetivos que ha conferido a su existencia y dan sentido a sus luchas. Ahora bien, pensar la equidad implica pensar a cada sujeto (en uso de su libertad) con posibilidades de realizar las acciones necesarias para alcanzar los fines que se ha propuesto, con unas condiciones en el sistema que atenúen o remueven los obstáculos que se presenten para alcanzar este propósito, sin afectar a los demás en sus posibilidades. En palabras de López (2005)

Desde esta perspectiva, la noción de equidad tiene un carácter eminentemente político. Por un lado, porque lleva implícita una valoración ética en su definición, al exigir una toma de posición sobre cuál es la igualdad estructurante que se define como horizonte. Por el otro, porque en tanto la equidad implica la búsqueda de la igualdad, esta igualdad fundamental que define los criterios de equidad no debe ser pensada como una situación dada, posible de ser mensurada en un momento específico, sino como un proyecto, un principio de organización que estructura el devenir de una sociedad. (pp. 68-69).

Estos planteamientos, desde posiciones filosóficas y políticas sobre la equidad, se materializan en los discursos de la política educativa. En estos se afirma la necesidad de garantizar que todos los sujetos tengan oportunidades y óptimas posibilidades de acceso, permanencia y éxito en el sistema educativo, sin distinción alguna. Las maneras como se ha materializado esta idea de equidad en la política educativa son las que hemos problematizado en este documento, ya que creemos que el concepto de equidad se ha trivializado en el propósito de hacerlo operativo en la política del sistema educativo. Reconocemos que en torno a estos tres asuntos asociados a la equidad, la política educativa se ha enfocado en: (1) el acceso como el ingreso al sistema, con el fin de asegurar cierto nivel de educación que se considera esencial para garantizar un mínimo de integración social, y no como brindar un lugar en el sistema al que llega reconociendo sus características individuales y su porvenir (la exclusión ${ }^{3}$ arrojaría manifestaciones de inequidad en el sistema); (2) la permanencia como retención en el sistema y no como igualdad de oportunidades para participar y actuar, con sentido, de las prácticas en el sistema educativo (privilegios, postergaciones o prácticas universales sin sentido para los sujetos son evidencias de inequidad); y (3) el éxito como resultado final individual, pero igual al de los demás y no como logro colectivo y constitución de intersubjetividades para posibilitar nuevas visiones del yo, del otro y del mundo (clasificación de los sujetos por los resultados de aprendizaje obtenidos frente a estándares universales serían manifestaciones de inequidad).

3 El lector interesado en un análisis de la igualdad, la equidad y la (in)exclusión en el currículo de matemáticas desarrollado por Edumadys puede remitirse a García y Valero (2013). 
Teniendo en cuenta lo anterior, pensar en mecanismos que garanticen la equidad en el sistema educativo implica, a nuestro entender, que el punto de partida deberá ser un conocimiento profundo, tanto de los infantes y jóvenes, como de los contextos en que se encuentran inmersos, de tal manera que se pueda responder, al menos parcialmente, preguntas como: ¿Quiénes son los infantes y jóvenes?, ¿cómo llegaron a ese contexto?, ¿qué momento histórico y social les correspondió vivir?, ¿cuáles son sus esperanza y anhelos?, entre otras. Es necesario entonces, al formular propuestas educativas, plantearse y responderse estas preguntas, tomando distancia de los lugares comunes en los que han sido formuladas y abordadas, lo que quizá dé paso a una reinvención del currículo en general y, para nuestro caso, de lo que pudiera acontecer en las aulas de matemáticas.

Una primera implicación al aproximarnos y reflexionar en torno a preguntas como las anteriores nos convoca a considerar la realidad social, cultural y política de nuestros estudiantes, antes que a aceptar una realidad homogeneizada y estandarizada por el poder hegemónico. Reconocemos que el primer paso para contribuir con condiciones de igualdad y equidad en prácticas educativas es no asumir los intereses particulares (establecidos desde las estructuras de poder) como si fuesen los intereses generales de la colectividad, así como no permitir que se imponga el bien individual como si fuese el bien común. Por ello, hemos comprendido que en el marco de las prácticas con las matemáticas que promovemos en las aulas de clase debemos reconocer y priorizar los intereses, metas y objetivos con los que nuestros infantes y jóvenes confieren sentido a su existencia en el marco de los contextos en los que van constituyendo sus subjetividades. De igual modo, aceptamos que desde la escuela contribuimos en esta constitución de subjetividades e intersubjetividades a través de los sistemas de razón que promovemos en ella (Salazar, Mancera y Camelo, 2013).

Teniendo en cuenta estas consideraciones y el objetivo de este artículo, la pregunta que nos planteamos es: desde las posturas que hemos asumido como colectivo que propende por el giro político en educación matemática en el contexto colombiano, ¿cómo podrían entenderse las posibilidades de acceso, permanencia y éxito a las prácticas con las matemáticas en la escuela? Así, a continuación, y solo a manera de reflexión, daremos cuenta de cada una de esas posibilidades desde nuestras propias prácticas pedagógicas e investigativas.

\section{Pensar en el acceso es más que llegar, implica ser reconocido al acceder}

Teniendo en cuenta las ideas del apartado anterior, pensamos en lo que podría significar el acceso de los estudiantes a las prácticas con las matemáticas en el aula de clase, desde el principio del reconocimiento de la diferencia y la diversidad. Pensar sobre los mecanismos que garantizan tal acceso implica no solo que los estudiantes puedan entrar en contacto 
con las matemáticas, sino que es en su experiencia humana que se logran integrar estas prácticas para construir comprensiones del mundo, nuevas o más complejas. Esto posibilitaría reconsiderar sus porvenires y abriría las puertas a la integración social; por ello, nuestra experiencia en prácticas pedagógicas y prácticas investigativas ha incorporado la caracterización del contexto y de los infantes y jóvenes, a partir de tratamientos sistemáticos y profundos tanto de sus narrativas y producciones artísticas, como de documentos locales e institucionales.

En consonancia con lo anterior, en nuestro trabajo planteamos comenzar por considerar quiénes son los infantes, los jóvenes y su disposición para participar en prácticas políticas o en acciones que conduzcan a comprender y transformar situaciones que socialmente resultan relevantes para su comunidad. En esta tarea de reconocimiento, hemos encontrado niños, niñas y jóvenes inquietos por situaciones de su entorno que les producen inconformismo e insatisfacción y que convocan su disposición e intenciones para la acción conjunta, sin evidenciar intereses en una acción política explícita o en una vinculación o identificación con grupos políticos.

Con estos grupos de infantes y jóvenes, nuestras experiencias han sido diversas, pues los jóvenes que encontramos en las escuelas, a quienes hemos denominado -retomando a Skovsmose, Scandiuzzi, Valero y Alrø (2011) - como de frontera, expresan preocupaciones de distinta naturaleza. Se trata de estudiantes que tienen la posibilidad de contrastar sus condiciones actuales de vida con otras, en particular con las de grupos socialmente hegemónicos, lo que arroja primeras manifestaciones de inequidad en el sistema. En las experiencias con grados sexto y séptimo hemos encontrado preocupaciones que comprometen condiciones más particulares de sus experiencias de vida, mientras en grado octavo estas se refieren a situaciones de impacto o afectación de un colectivo mayor, que puede ser el barrio o la localidad (Salazar, Mancera y González, 2013). Esta diversidad de preocupaciones encontradas en los jóvenes de las escuelas en las que hemos desarrollado nuestro trabajo nos pone de presente las afirmaciones encontradas en Kriger (2014) al referirse a la no homogeneidad de esta población:

... los jóvenes no componen un sujeto colectivo homogéneo, sino que son muy diferentes entre sí. Asimétricamente diferentes, en correspondencia con las desigualdades de clase, étnicas, culturales, además extremas en América Latina. Por eso mismo es tan complejo pero también tan relevante-casi indispensable- poder encontrar no solo rasgos comunes, sino vivencias, horizontes, experiencias generacionales en común, compartidos o a compartir, [sic] que atraviesen y vertebren esta apuesta por seguir viviendo juntos (Touraine, 1997), esta variante al fin de la "aventura obstinada" (Badiou y Truong, 2012) que seguimos Ilamando proyecto común. (p. 593). 
La experiencia que llevamos a cabo con el grupo de sexto grado, con infantes de 10 años de edad en promedio, ubicados en un barrio popular y con una cultura de aula tradicional, giró en torno a la pregunta "¿Cómo me relaciono con los lugares que habito y qué posibilidades de realización encuentro en ellos?". Después del trabajo con los estudiantes, el escenario lo denominamos "Un día de vacaciones en mi ciudad". Esta experiencia surgió a partir de las manifestaciones de desaliento de los estudiantes ante la llegada de sus vacaciones, pues la gran mayoría de ellos y sus familias no contaban con recursos para salir de la ciudad, por lo cual, no los motivaba la idea de disfrutar de un periodo de vacaciones.

Al escuchar a los estudiantes, encontramos un asunto que por una parte podría articular sus intenciones-disposiciones para la acción, y por otra, podría promover prácticas con las matemáticas que aportaran a la resignificación de los lugares que habitaban. Decidimos reconstruir con ellos las opciones que encontraban en su ciudad para la diversión y las vacaciones, pues estos estudiantes, a pesar de vivir en ella, no la conocían. Cabe aclarar que la localidad en la que habitaban estos infantes y donde se encuentra la escuela está ubicada en zonas periféricas de Bogotá y se caracteriza por la concentración de comercio informal o semiformal, por lo cual, las prácticas culturales de las familias están relacionadas con esta característica del espacio físico cercano, y un buen número de estudiantes participaban en las prácticas económicas de su familia y la localidad.

La intención fundamental del proyecto era recrear la ciudad como lugar que habitan los estudiantes, considerando las posibilidades que les ofrece para su desarrollo y para otras prácticas culturales. Para nosotros como grupo de investigación era claro que el proyecto se presentaba como una posibilidad para que los miembros de esta comunidad (no solo los estudiantes sino las familias) pudieran comprender la importancia de su relación con el espacio en el que habitan, comprenderlo como espacio localizable, concreto, cambiante y diferenciado. En ese momento los planteamientos de Segrelles (2002), en los que rescata algunas ideas de Dollfus, nos llevaron a comprender que: (1) el soporte del sistema de relaciones sociales que se establecen en una comunidad está determinado no solo por elementos del medio físico, sino también por las características de las sociedades que ordenan ese espacio y encarnan un tejido histórico que constituye la civilización, y (2) para resignificar las prácticas con las matemáticas que involucran el espacio, no basta con interpretar las formas geométricas o las prácticas de medición, sino que es necesario interpretar lo que representan culturalmente estos espacios para los sujetos.

Este escenario para las prácticas con las matemáticas condujo a la construcción de planes de vacaciones para un día en la ciudad, que incluían información sobre costos, vías de acceso, características del lugar, su importancia para la ciudad y para las comunidades, actividades

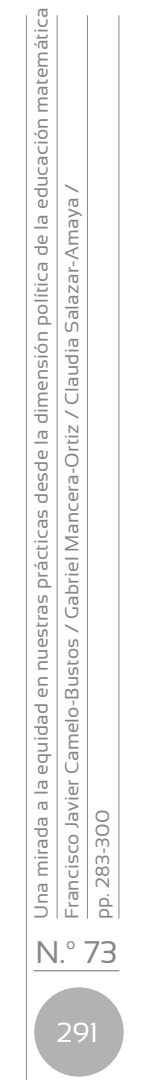


que se podían realizar, alimentación y opciones de transporte. El trabajo fue presentado a la comunidad de la escuela en una "feria de agencias de viaje" que organizaron los estudiantes. Si bien no es propósito de este documento presentar con detalle y profundidad las prácticas con las matemáticas que se propician, parece necesario aclarar que en estas no se trabajan con modelos matemáticos descontextualizados. Por el contrario, se analizan las limitaciones de modelos matemáticos establecidos con un cierto número de variables para dar cuenta de las situaciones o fenómenos de la realidad, considerando nuevas variables que reflejan su complejidad y que aparecen en el análisis a partir de la experiencia humana de los sujetos en esas situaciones.

Un ejemplo de lo anterior tiene que ver con las discusiones acerca de los mapas y las formas de acceder a algún lugar en la ciudad. Esta práctica de localizar no podía analizarse solo desde el modelo matemático en abstracto, esto es, usando conceptos de la geometría plana de Euclides, como el de menor distancia entre dos puntos, pues esta idea no es funcional para pensar la mejor forma de llegar de un lugar a otro en la ciudad. Esta relación en el espacio está mediada por la idea de distancia mental que construye el sujeto en el mundo vivido (no en el objetivo). La idea de distancia mental involucra otras variables, como la facilidad para recorrer esta distancia, la dificultad para trazar ese itinerario urbano o incluso la seguridad que ofrezca el trayecto.

Esperamos que la descripción sucinta de la experiencia anterior sea ejemplarizante en cuanto al reconocimiento de los siguientes aspectos: (1) relaciones entre los sujetos, los contextos y las prácticas culturales en las que están inmersos; (2) una función política para las prácticas con las matemáticas; (3) la experiencia humana como fuente de otro tipo de conocimiento que se relaciona con las matemáticas y nutre sus prácticas, posibilitando concebir nuevos mundos. Esta manera de ver las prácticas con las matemáticas, inmersas en la experiencia humana, es la que consideramos permite a los estudiantes entrar en contacto con ellas y brindarles acceso en el marco de las ideas de equidad que planteamos.

\section{Pensar en la permanencia es más que estar, es encontrar sentido para permanecer}

Desde los actuares, propósitos y perspectivas en los que nos enmarcamos como colectivo de trabajo, reflexionar sobre la idea de la permanencia en nuestras prácticas de aula con las matemáticas, como igualdad de oportunidades para participar y actuar con sentido en ellas, nos ha implicado considerar también aspectos que tradicionalmente se denominan bajo el rótulo del desinterés y la falta de motivación de los estudiantes. Reconocemos por una parte que para lograr que los estudiantes se mantengan, 
es necesario que acepten la invitación de participar, actuar y quedarse; por otra, que el desinterés se asocia como uno de los problemas en el aprendizaje de las matemáticas que día a día toma mayor fuerza.

Este interés es reportado en García, Valero y Mancera (2009), con estudiantes de séptimo grado de un colegio público ubicado en la periferia de la ciudad (al suroriente), que viven en condiciones sociales y económicas adversas. Con este grupo de estudiantes buscamos, entre otros hechos, reflexionar sobre el desinterés por el aprendizaje desde el punto de vista de ellos, circunstancia que se asocia con las intenciones del aprendizaje (Skovsmose, 1994) en la medida en que estas "se basan en un conjunto complejo de disposiciones que pueden guiar al estudiante en muchas direcciones diferentes, no todas paralelas a la dirección que el profesor hubiera podido esperar" (Skovsmose, 1994, p. 202).

Particularmente en el caso de clase reportada y analizada en este trabajo, observamos que las intenciones también podrían estar relacionadas con circunstancias como las que exponen García et al. (2009):

» La presión de la familia, como es el caso de Edgar Alfonso: "porque me toca... porque me toca... o sea es que yo no es que quiera el estudio ni nada... no me agrada" (p. 61).

» La esperanza de ser alguien en la vida, como lo expone José: "aprender para ser alguien en la vida" (p. 61).

»La expectativa de tener trabajo y conseguir "algo", como lo expresa Viviana: "una persona que pueda ser y tener un mejor futuro y no quedarse así sino tener un buen estudio para tener un desempeño trabajando y conseguir algo" (p. 61).

"La ilusión de un futuro concreto, que solucione las situaciones económicas complejas que se viven, como lo menciona Yenny: "sí uno estudiando puede tener más opciones de trabajar que si usted no estudia pues no hace nada...mejor dicho, entonces solo con el bachillerato y cualquier curso ... algo ... lo reciben en cualquier empresa" (p. 62).

Al respecto, y siguiendo las ideas de Skovsmose, en Camelo, Mancera, García y Romero (2008) señalamos que:

» Es necesario conformar un proyecto que posibilite que el proceso de enseñar y aprender, ofrezca las posibilidades a estudiantes y profesores para encontrar las razones del porqué y para qué del propósito del proceso educativo, es decir, que los estudiantes puedan claramente conocer su significado. (Camelo et al., p. 1)

Teniendo como marco la anterior consideración, planteamos, inicialmente, la necesidad de crear escenarios que permitan constituir una situación en la que el proceso educativo pueda encarnarse para dar 
significado a las actividades que los estudiantes deben desarrollar. Con base en las investigaciones realizadas y en los trabajos de pregrado y maestría desarrollados en el seno del colectivo, esta idea de escenario ha sido recontextualizada como herramienta teórica y analítica, estableciendo la noción de escenarios y ambientes de aprendizaje de las matemáticas (García, Valero y Camelo, 2013). De esta manera, para la creación de dichos escenarios, y con la intención de propender por una participación y actuación con sentido de los estudiantes en prácticas en el sistema educativo, hemos asumido seis dimensiones que integran aspectos relevantes para una mejor comprensión de la complejidad social y política que constituyen las aulas de matemáticas. Dichas dimensiones son: (1) las intenciones y los porvenires de los estudiantes como una realidad sociopolítica; (2) la construcción de la subjetividad social y el reconocimiento de las diversidades culturales de los estudiantes; (3) la racionalidad de las matemáticas escolares; (4) la materialidad para el aprendizaje; (5) la interacción dialógica entre estudiantes, y entre estudiantes y profesor en el aula, y (6) las políticas de escolarización de las matemáticas.

En consonancia con las anteriores ideas, en el colectivo hemos buscado tomar una distancia crítica en relación con la organización estándar de las prácticas educativas tradicionales. El propósito es pensar un currículo que haga posible:

... una escuela abierta, de contornos mucho más difusos que los del modelo tradicional. De esta manera la relación de los contenidos del aprendizaje con la posición de los niños implica identificar las múltiples y complejas relaciones con sus vidas sociales. (Camelo et al., 2008, p. 2).

Así, y teniendo como telón de fondo el anterior panorama, desde nuestra mirada, el currículo debe permitir no solo involucrar la intencionalidad del aprendizaje de los estudiantes y conjugar su porvenir, sino que debe procurar prácticas que favorezcan la participación con sentido.

Los escenarios y ambientes educativos de aprendizaje, a la luz de estas consideraciones, nos han permitido, entre otras circunstancias, explorar aspectos inherentes a la permanencia tales como: la microcultura de la clase, las tensiones que se gestan en la creación de los escenarios, el uso de distintos lenguajes y el reconocimiento de diversas trayectorias de aprendizaje.

Teniendo en cuenta lo anterior, nos fue natural aceptar que concebir la realidad (social, histórica, política y económica) de las intenciones de los estudiantes de participar en el aprendizaje de las matemáticas implica una conexión (fuerte y clara) entre su microcontexto y su amplio macrocontexto sociopolítico, como miembros de una sociedad particular en un tiempo histórico dado (Valero, 2002). Este hecho también nos permitió aceptar que, para lograr la participación y actuación de los estudiantes, 
debíamos prestar atención al constructo disposiciones-intenciones-acciones de los estudiantes (Skovsmose, 1994), pues al explorarlo podríamos encontrar en ellos razones para aprender y sentidos para permanecer en las prácticas con las matemáticas en el aula de clase.

\section{Pensar el éxito es más que alcanzar individualmente, implica conquistar colectivamente}

Para reflexionar lo que podría considerarse como el éxito en nuestras prácticas de aula con las matemáticas, desde la perspectiva que hemos venido desarrollado, tendríamos que tener en cuenta-como hemos venido insistiendo a lo largo de este texto- prioritariamente aspectos inherentes al ser humano. Lo anterior ya que debemos recoger asuntos que posibiliten incorporar unos entendimientos de la subjetividad más contemporáneos y abandonar los rasgos de la subjetividad moderna que han sido hegemónicos en nuestro campo de trabajo, para producir un fuerte centramiento en el contenido y su organización desde un punto de vista racional.

Tales interpretaciones de la subjetividad más contemporáneas las encontramos en nuestras investigaciones, al plantear que en las prácticas de aula, lo social se constituye en un asunto esencial por cuanto abre posibilidades para incorporar (incorporándonos) a los otros en nuestras comprensiones del mundo, las cuales toman referencia en las problemáticas, reinterpretaciones y posibilidades que se dan en los contextos (sociales, culturales, históricos, políticos, etc.) en que se ubican nuestros estudiantes. Así, y tal y como lo plantea Valero (2006, p. 8), lo político no queda por fuera de esta reflexión, pues

\footnotetext{
En toda práctica social hay elementos de poder involucrados. El poder es una característica de las relaciones sociales y por tanto está presente en y entre todos los grupos humanos, instituciones y sociedades, tanto en la vida pública como en la privada. El poder se expresa en todas las relaciones, instituciones y estructuras que están implicadas en la producción y reproducción de la vida de las sociedades y comunidades. El poder crea y condiciona todos los aspectos de nuestra vida y está en el centro del desarrollo de problemas colectivos y de los modos de su resolución. La educación matemática como práctica social de gran importancia en el mundo actual no se escapa de lo político.
}

Reconocemos que toda forma de conocer instaura una cierta manera de ser y que las maneras de ser conllevan formas de conocer. Por ello, los argumentos o discursos que generamos sobre el éxito y sobre cuáles son los resultados esperados están permeados por estas formas que concebimos de conocer y ser en el mundo. Nuestra apuesta es por formas de conocer con las matemáticas que instauran maneras de ser más humanas y seres humanos que conozcan de otros modos el mundo, ayudados de prácticas con las matemáticas. 
Así, en lugar de prestar atención a aspectos puramente cognitivos, por ejemplo, hemos optado por enfocarnos en la experiencia. Lo anterior, debido a que consideramos que la participación en actividades colectivas posibilita a nuestros infantes y jóvenes poner en debate sus ideas personales e incorporar (incorporándose) al otro en las comprensiones del mundo y de las problemáticas que se identifican y se decide abordar. En un sentido arendtiano, planteamos que la experiencia podría interpretarse como un movimiento entre la individualidad y la colectividad como posibilidad de ser con el otro, sin anularse y sin quedar sumergidos en la colectividad, alienándonos; al mismo tiempo que se toma distancia del colectivo y se forma un criterio propio que tiene en cuenta e incorpora las narraciones del otro.

Bajo el criterio de la experiencia, en casi la totalidad de los proyectos que hemos desarrollado, podemos identificar una preocupación porque los estudiantes nos ayuden a comprender sus contextos y los reinterpreten con base en saberes matemáticos que se construyen como parte de la investigación que planteamos desarrollar conjuntamente. Lo anterior, como parte de una reinterpretación del currículo que descentra los contenidos como organizador del plan de estudios y da lugar a un reconocimiento de la subjetividad.

En Camelo, Mancera y Perilla (2016) y Mancera, Camelo y Perilla (2016), por ejemplo, reportamos cómo un grupo de estudiantes de grado undécimo de un colegio público de Bogotá (ubicado en una de las localidades con mayor densidad demográfica donde los índices de pobreza e indigencia son altos, lo que redunda en problemas de inseguridad acentuados), organizados en subgrupos de 4 y 5 integrantes, discutieron problemáticas relacionadas con la telefonía móvil en Colombia, pues el año anterior a la creación de este ambiente de modelación matemática, se promulgó una ley que cambiaba las dinámicas de este servicio en el país. Durante el desarrollo del ambiente los estudiantes fueron planteando problemáticas que ellos mismos habían vivenciado como usuarios de este tipo de tecnologías de la comunicación y pudieron elaborar comprensiones más profundas y fundamentadas de lo que ocurría, lo que dio lugar a interpretaciones con soportes matemáticos. En ese contexto fue posible observar que, inicialmente, muchos de ellos compartían apreciaciones sobre tales problemáticas que devienen de creencias populares. Estas opiniones fueron variando gracias a la búsqueda y el análisis de información sobre el tema, de manera que la experiencia les posibilitara espacios de reflexión y discusión donde cada uno aportaba desde sus marcos de referencia, a la vez que los iba recontextualizando gracias al debate con los otros y a la incorporación de informaciones que entraban al aula.

De otro lado, hemos buscado comprender y promover que cuando un estudiante o profesor narra su experiencia, esta innegablemente está plagada de las narraciones que otros han desarrollado antes y que contribuyen a la 
constitución de cada uno de los participantes de la clase. Este reconocimiento del otro en cada uno implica entender que estamos en constante constitución y que no se pretende comprensiones terminadas ni finales, pues lo interesante es evidenciar que gracias a las discusiones construimos interpretaciones cada vez más complejas. Así, la vieja idea que se persigue en las clases de matemáticas acerca de conceptualizaciones acabadas es reemplazada por una constante búsqueda de la completitud, entendida como la oportunidad de profundizar cada vez más en nuestras interpretaciones.

Lo anterior nos ayuda, también, a comprender que las posibilidades del éxito pueden interpretarse en las interacciones que se dan entre los sujetos, pues en la clase de matemáticas este no debe estar mediado únicamente por un orden cognitivo que dé cuenta de los aprendizajes conceptuales, sino que debe dar entrada a las intenciones de aprendizaje. Estas permiten entonces incorporar contextos socialmente relevantes que nos interesa comprender, y por tanto nos impulsan a profundizar nuestras interpretaciones, dándonos explicaciones al porqué estamos aquí y hacia dónde queremos dirigir esfuerzos que nos hagan considerar porvenires.

Por otra parte, en uno de nuestros primeros proyectos desarrollamos una serie de escenarios con los que pretendíamos explorar el alcance y las posibilidades de trabajo al reinventar el currículo de matemáticas (García et al., 2008), descentrando la tensión sobre los contenidos matemáticos y dando lugar a que lo social los antecediera, por lo que estos no se definían con anterioridad, lo que ha caracterizado todos nuestros proyectos de investigación.

En uno de tales escenarios, con un grupo de estudiantes de séptimo grado (Camelo, et al., 2010), detectamos que los refrigerios que eran repartidos a la mitad de la jornada escolar resultaban demasiado dulces para los estudiantes de los grados superiores y, por tal razón, los botaban al cesto de la basura luego de probarlos. Los estudiantes de grado séptimo, además, luego de analizar un poco la situación y hacer consideraciones desde el sentido común, infirieron que el exceso de azúcar en los refrigerios podría estar provocando que algunos de ellos fueran más activos de lo esperado, lo que generaría problemas de convivencia, pues el dulce es una fuente de energía extra. Con estas dos consideraciones, el grupo (estudiantes y profesor) acordó realizar una investigación sobre las necesidades nutricionales que ellos tenían y contrastarlas con los alimentos recibidos, pues comenzaron a sospechar que no eran los adecuados y que, quizá, los políticos de turno ofrecían tales alimentos sin tener en cuenta criterios nutricionales.

El proyecto se desarrolló por varias semanas y los estudiantes alcanzaron, incluso, a analizar y proponer distintos tipos de menú para que los prepararan en sus propios hogares, pues resultaron involucrados al notar que era posible que ellos propusieran cambios, en sus hogares, en beneficio de su nutrición y la de su familia. 
Así, consideramos que el éxito en el aula de matemáticas no radica en el alcance de logros individuales en relación con contenidos previamente establecidos, sino que implica, después de acceder a las prácticas con las matemáticas, sintiéndose reconocido y habiendo encontrado razones para permanecer en ellas, conquistar colectivamente sentidos para actuar en el mundo y ser con otros.

\section{Entrecierre de la reflexión}

Las consideraciones que hemos presentado aquí, producto de nuestras experiencias pedagógicas e investigativas en el campo de la educación matemática, nos Ilevaron a cuestionar las maneras como tradicionalmente el sistema educativo colombiano ha promovido, a través de la política educativa, mecanismos para garantizar la equidad.

Como podemos apreciar, desde nuestras comprensiones, la equidad en las aulas de matemáticas debería relacionarse con formas de ser más humanas y centradas en un entendimiento de la subjetividad que incorpora posibilidades de actuar y ser con otros. Así, en la medida en que se encuentran razones para debatir y discutir asuntos de relevancia social, los estudiantes encuentran oportunidades para analizar lo que está aconteciendo, basados en sus experiencias, y dar paso a nuevas formas de entenderse en el contexto en que están inmensos con el fin de dar cuenta del porqué se está en tales condiciones.

De esta manera, planteamos que podrían proponerse alternativas de cambio, partidas del seno de la misma comunidad, que se convierten en conquistas colectivas. En este sentido, el acceso, la permanencia y el éxito, como los hemos problematizado en este documento, se constituyen en pilares desde los que pudiera aportarse a la equidad desde/para la educación matemática.

\section{Referencias}

Arroyo, M. G. (2015). O direito à educação e a nova segregação social e racial: Tempos insatisfatórios? Educação Em Revista, 31(3), 15-47.

Bracho, T. y Hernández, J. (septiembre de 2009). Equidad educativa: avances en la definición de su concepto. Ponencia presentada durante el X Congreso Nacional de Investigación Educativa. Veracruz, México.

Camelo, F.; Mancera, G.; García, G. y Romero, J. (2008). Reinventando el currículo y los escenarios de aprendizaje de las matemáticas, de la espacialidad. Un estudio desde la perspectiva de la educación matemática crítica. Bogotá: Fondo Editorial Universidad Pedagógica Nacional. 
Camelo, F., Mancera, G., Romero, J., García, G. y Valero, P. (abril de 2010). The importance of the relation between the socio-political context, interdisciplinarity and the learning of mathematics. Proceedings of the Sixth International Mathematics Education and Society Conference, 1, 199-208.

Camelo, F., Perilla, W. y Mancera, G. (2016). Prácticas de modelación matemática desde una perspectiva socio crítica con estudiantes de grado undécimo. Revista Latinoamericana de Etnomatemática, 9(2), 67-84.

Congreso de la República de Colombia, (2015). Plan Nacional de Desarrollo 2014-2018 "Todos por un nuevo país".

García, G., Valero, P. y Camelo, F. (2013). Escenarios y ambientes educativos de aprendizaje de las matemáticas. Constitución de subjetividades en educación matemática elemental. En G. García, P. Valero, C. Salazar, G. Mancera, F. Camelo y J. Romero (eds.). Procesos de inclusión/exclusión, subjetividades en educación matemática. Bogotá: Fondo Editorial Universidad Pedagógica Nacional.

García, G., Valero, P., \& Mancera, G. (2009). Condiciones y oportunidades para configurar en el aprendizaje de las matemáticas la subjetividad en procesos de socialización. En G. García, P. Valero, F. Camelo, G. Mancera, J. Romero, G. Peñaloza y S. Samaca (eds.). Escenarios de aprendizaje de las matemáticas: Un estudio desde la perspectiva de la educación matemática crítica. Bogotá: Fondo Editorial Universidad Pedagógica Nacional.

García, G., Valero, P., Camelo, F., Mancera, G., Romero, J., Peñaloza, G. y Samaca, S. (2009). Escenarios de aprendizaje de las matemáticas: un estudio desde la perspectiva de la educación matemática crítica. Bogotá: Fondo Editorial Universidad Pedagógica Nacional.

García y Valero (2013). De la igualdad, la equidad y la (in)exclusión en el currículo de matemáticas: una revisión en el contexto colombiano. En G. García, P. Valero, C. Salazar, G. Mancera, F. Camelo y J. Romero. Procesos de inclusión/exclusión, subjetividades en educación matemática (pp. 115-145). Bogotá: Fondo Editorial Universidad Pedagógica Nacional.

Kriger, M. (2014). Politización juvenil en las naciones contemporáneas. El caso argentino. Revista Latinoamericana de Ciencias Sociales, Niñez y Juventud, 12(2), pp. 583-596.

López, N. (2005). Equidad educativa y desigualdad social. Desafíos de la educación en el nuevo escenario Latinoamericano. Buenos Aires: IIPE-Unesco.

Mancera, G.; Camelo, F. y Perilla, W. (julio de 2016). Modelación matemática desde la perspectiva sociocrítica con estudiantes de secundaria: posibilidades y retos. En Educação Matemática na Contemporaneidade: desafios e possibilidades. São Paulo: Sociedade Brasileira de Educação Matemática. 
Said, S. y Kriger, M. (2014). Subjetivación política y educación popular: la noción del diálogo en Ranciere y Freire como aporte a la reflexión teórico-metodológica sobre bachilleres populares. Questión, 1(42), 405-418.

Salazar, C.; Mancera, G. y González, M. (septiembre de 2013). Ambientes de aprendizaje, inclusión e intersubjetividad. En Memorias VII Congreso Iberoamericano de Educación Matemática, Montevideo.

Salazar, C.; Mancera, G. y Camelo, F. (2013). Ambientes de aprendizaje e intersubjetividad. En P. Valero, G. García, C. Salazar, G. Mancera, F. Camelo y J. Romero. Procesos de inclusión/exclusión: subjetividades en educación matemática. Bogotá: Fondo Editorial Universidad Pedagógica Nacional.

Segrelles, J. (2002). Geografía humana. Fundamentos, métodos y conceptos. Alicante: ECU. Universidad de Alicante.

Skovsmose, O. (1994). Towards a philosophy of critical mathematics education. Dordrecht, Boston: Kluwer Academic Publishers.

Skovsmose, O.; Scandiuzzi, P.; Valero, P. y Alrø, H. (2011). Aprender matemáticas en una posición de frontera: los porvenires y la intencionalidad de los estudiantes en una favela brasilera. Revista Educación y Pedagogía, 23(59), 103.

Valero, P. (2002). The myth of the active learner: From cognitive to socio-political interpretations of students in mathematics classrooms. En P. Valero y O. Skovsmose (eds.), Proceedings of the Third International Conference on Mathematics Education and Society (2. ${ }^{\text {a ed., }}$ vol. 2). Copenhagen: Center for Research in Learning Mathematics.

Valero, P. (2006). ¿De carne y hueso? La vida social y política de la competencia matemática. Memorias de Foro Educativo Nacional. Bogotá: Ministerio de Educación Nacional.

Valero, P. y Skovsmose, O. (eds.) (2012). Educación matemática crítica. Una visión sociopolítica del aprendizaje y la enseñanza de las matemáticas. Bogotá: Una Empresa Docente-Uniandes. 